\title{
The Application of the Vector Space Theory to the Limit of Number Sequence
}

\author{
Xingxiang Liu \& Na Feng \\ College of Mathematics and Computer Science, Yan'an University, Yan'an 716000, China
}

Tel: 86-0911-285-9468Ｅ-mail: 1xx6407@163.com

\begin{abstract}
This article studied the application of vector space theory in the mathematical analysis in order to enhance the teaching and researching levels of the mathematical analysis and strengthen the teaching and researching levels of the higher algebra.
\end{abstract}

Keywords: Vector space, Base, Limit, Recurrence relations

The mathematical analysis and the higher algebra are two important professional basic lessens in various specialties of the college mathematical department. To a certain extent, any profound mathematical methods are to transform the complex mathematical objective to relative contents of the mathematical analysis and the higher algebra. Though these two lessons are different in the solving method, but they are closely associated and combined each other in many aspects.

The concepts and ideas that utilize the vector space to solve problems (Mi, 2002, p.66-69 \& Song, 2004, p.100-101) still have many deficiencies, so in this article, we will further discuss this problem.

Definition 1: suppose $_{V}=\left\{\left\{u_{n}\right\} \mid u_{n}=\sum_{k=1}^{l} a_{k} u_{n-k}, a_{k} \in F(k=1,2, \cdots, l), n \geq l+1\right\}$.

Regulation: (1) $\forall\left\{u_{n}\right\},\left\{v_{n}\right\} \in V$, so, $\left\{u_{n}\right\}+\left\{v_{n}\right\} \in V$

(2) $\forall k \in F, \forall\left\{u_{n}\right\} \in V$, so, $k\left\{u_{n}\right\}=\left\{k u_{n}\right\} \in V$

So, V about Regulation (1) and (2) is the vector space on the number field F.

Lemma 1: suppose $V=\left\{\left\{u_{n}\right\} \mid u_{n}=\sum_{k=1}^{l} a_{k} u_{n-k}, a_{k} \in F(k=1,2, \cdots, l), n \geq l+1\right\}$ and $\mathrm{V}$ about Regulation (1) and (2) is the vector space on the number field $\mathrm{F}$, and $x^{l}=\sum_{k=1}^{l} a_{k} x^{k-1}$ has $l$ single roots, $q_{1}, q_{2}, \cdots, q_{l}$, so

(1) $\mathrm{V}$ is $l$ dimensional vector space on $\mathrm{F}$

(2) $\left\{q_{1}^{n}\right\},\left\{q_{2}^{n}\right\}, \cdots,\left\{q_{l}^{n}\right\}$ is a set of base of $\mathrm{V}$

(3) Suppose $u_{n}=\sum_{k=1}^{l} b_{k} q_{k}^{n}$, so according to the initial condition $u_{1}, u_{2}, \cdots, u_{l}$, we can confirm $b_{k}(k=1,2, \ldots, l)$ and obtain the recurrence relations $u_{n}=\sum_{k=1}^{l} b_{k} q_{k}^{n}$.

Certification: Because $x^{l}=\sum_{k=1}^{l} a_{k} x^{k-1}$ has $l$ single roots, and suppose $q_{1}, q_{2}, \cdots, q_{l}$. 
Because $q_{1}, q_{2}, \cdots, q_{l}$ has $l$ single roots, so $\left(\begin{array}{c}q_{1} \\ q_{1}^{2} \\ \vdots \\ q_{1}^{l}\end{array}\right),\left(\begin{array}{c}q_{2} \\ q_{2}^{2} \\ \vdots \\ q_{2}^{l}\end{array}\right), \cdots,\left(\begin{array}{c}q_{1} \\ q_{l}^{2} \\ \vdots \\ q_{l}^{l}\end{array}\right)$ are linear independent, i.e. the $\operatorname{rank}\left(\left(\begin{array}{c}q_{1} \\ q_{1}^{2} \\ \vdots \\ q_{1}^{l}\end{array}\right),\left(\begin{array}{c}q_{2} \\ q_{2}^{2} \\ \vdots \\ q_{2}^{l}\end{array}\right), \cdots,\left(\begin{array}{c}q_{l} \\ q_{l}^{2} \\ \vdots \\ q_{l}^{l}\end{array}\right)\right)=l$

Because the number sequence $\left\{u_{n}\right\}$ is only confirmed by former $l$ items $u_{1}, u_{2}, \cdots, u_{l}$, so the necessary and sufficient condition that $\left\{u_{n}^{(1)}\right\},\left\{u_{n}^{(2)}\right\}, \cdots,\left\{u_{n}^{(l)}\right\}$ are linear independent is that $\left(\begin{array}{c}u_{1}^{(1)} \\ u_{2}^{(1)} \\ \vdots \\ u_{l}^{(1)}\end{array}\right),\left(\begin{array}{c}u_{1}^{(2)} \\ u_{2}^{(2)} \\ \vdots \\ u_{l}^{(2)}\end{array}\right), \cdots,\left(\begin{array}{c}u_{1}^{(l)} \\ u_{2}^{(l)} \\ \vdots \\ u_{l}^{(l)}\end{array}\right)$ are linear independent. Suppose $_{F^{l}}=\left\{\left(\begin{array}{c}u_{1} \\ u_{2} \\ \vdots \\ u_{l}\end{array}\right) u_{i} \in F(i=1,2, \cdots, l)\right\}$, so for any $\left(\begin{array}{c}u_{1} \\ u_{2} \\ \vdots \\ u_{l}\end{array}\right) \in F^{l},\left(\begin{array}{c}u_{1} \\ u_{2} \\ \vdots \\ u_{l}\end{array}\right)$ can be linearly presented by $\left(\begin{array}{c}q_{1} \\ q_{1}^{2} \\ \vdots \\ q_{1}^{l}\end{array}\right),\left(\begin{array}{c}q_{2} \\ q_{2}^{2} \\ \vdots \\ q_{2}^{l}\end{array}\right), \ldots,\left(\begin{array}{c}q_{l} \\ q_{l}^{2} \\ \vdots \\ q_{l}^{l}\end{array}\right)$. So $\left\{u_{n}\right\}$ can be linear presented by $\left\{q_{1}^{n}\right\},\left\{q_{2}^{n}\right\}, \cdots,\left\{q_{l}^{n}\right\}$, so $\mathrm{V}$ is the $l$ dimensional vector space on $\mathrm{F}$, and $\left\{q_{1}^{n}\right\},\left\{q_{2}^{n}\right\}, \cdots,\left\{q_{l}^{n}\right\}$ is one set of base of V.

Because $\left\{u_{n}\right\}$ can be linear presented by $\left\{q_{1}^{n}\right\},\left\{q_{2}^{n}\right\}, \cdots,\left\{q_{l}^{n}\right\}$, so $u_{n}=\sum_{k=1}^{l} b_{k} q_{k}^{n}$, and according to the initial condition $u_{1}, u_{2}, \cdots, u_{l}$, we can confirm $b_{1}, b_{2}, \cdots, b_{l}$, so $u_{n}=\sum_{k=1}^{l} b_{k} q_{k}^{n}$.

Lemma 2: suppose $V=\left\{\left\{u_{n}\right\} \mid u_{n}=\sum_{k=1}^{l} a_{k} u_{n-k}, a_{k} \in F(k=1,2, \cdots, l), n \geq l+1\right\}$ and $\mathrm{V}$ about Regulation (1) and (2) is the vector space on the number field $\mathrm{F}$, and $q_{1}$ is $l$ multiple root of $x^{l}=\sum_{k=1}^{l} a_{k} x^{k-1}$, so

(1) $\mathrm{V}$ is $l$ dimensional vector space on $\mathrm{F}$

(2) $\left\{q_{1}^{n}\right\},\left\{n q_{1}^{n}\right\},\left\{n^{2} q_{1}^{n}\right\}, \cdots,\left\{n^{l-1} q_{1}^{n}\right\}$ is a set of base of V

(3) Suppose $u_{n}=\sum_{k=1}^{l} b_{k} n^{k-1} q^{n}$, so according to the initial condition, the values of $u_{1}, u_{2}, \cdots, u_{l}$, we can $\operatorname{confirm} b_{k}(k=1,2, \ldots, l)$ and obtain the recurrence relations $u_{n}=\sum_{k=1}^{l} b_{k} n^{k-1} q^{n}$.

Certification: Because $q_{1}$ is $l$ multiple root of $x^{l}=\sum_{k=1}^{l} a_{k} x^{k-1}$, so $\left(\begin{array}{c}q_{1} \\ q_{1}^{2} \\ \vdots \\ q_{1}^{l}\end{array}\right),\left(\begin{array}{c}q_{2} \\ 2 q_{1}^{2} \\ \vdots \\ l q_{1}^{l}\end{array}\right),\left(\begin{array}{c}q_{2} \\ 2^{2} q_{1}^{2} \\ \vdots \\ l^{2} q_{1}^{l}\end{array}\right), \cdots,\left(\begin{array}{c}q_{l} \\ 2^{l-1} q_{1}^{2} \\ \vdots \\ l^{l-1} q_{1}^{l}\end{array}\right)$ are linear independent 
and the rank $\left(\left(\begin{array}{c}q_{1} \\ q_{1}^{2} \\ \vdots \\ q_{1}^{l}\end{array}\right),\left(\begin{array}{c}q_{2} \\ 2 q_{1}^{2} \\ \vdots \\ l q_{1}^{l}\end{array}\right),\left(\begin{array}{c}q_{2} \\ 2^{2} q_{1}^{2} \\ \vdots \\ l^{2} q_{1}^{l}\end{array}\right), \cdots,\left(\begin{array}{c}q_{l} \\ 2^{l-1} q_{1}^{2} \\ \vdots \\ l^{l-1} q_{1}^{l}\end{array}\right)\right)=r$

So $\left\{q_{1}^{n}\right\},\left\{n q_{1}^{n}\right\},\left\{n^{2} q_{1}^{n}\right\}, \cdots,\left\{n^{l-1} q_{1}^{n}\right\}$ are linear independent, and $\left\{q_{1}^{n}\right\},\left\{n q_{1}^{n}\right\},\left\{n^{2} q_{1}^{n}\right\}, \cdots,\left\{n^{l-1} q_{1}^{n}\right\} \in V$.

Because the number sequence $\left\{u_{n}\right\}$ is only confirmed by former $l$ items $u_{1}, u_{2}, \cdots, u_{l}$ of $u_{n}$, and when $\left\{u_{n}^{(1)}\right\},\left\{u_{n}^{(2)}\right\}, \cdots,\left\{u_{n}^{(l)}\right\}$ are linear independent, so $\left(\begin{array}{c}u_{1}^{(1)} \\ u_{2}^{(1)} \\ \vdots \\ u_{l}^{(1)}\end{array}\right),\left(\begin{array}{c}u_{1}^{(2)} \\ u_{2}^{(2)} \\ \vdots \\ u_{l}^{(2)}\end{array}\right), \cdots,\left(\begin{array}{c}u_{1}^{(l)} \\ u_{2}^{(l)} \\ \vdots \\ u_{l}^{(l)}\end{array}\right)$ are linear independent.

Suppose $F^{l}=\left\{\left(\begin{array}{c}u_{1} \\ u_{2} \\ \vdots \\ u_{l}\end{array}\right) \mid u_{i} \in F(i=1,2, \cdots, l)\right\}, \quad$ so $\quad$ for any $\left(\begin{array}{c}u_{1} \\ u_{2} \\ \vdots \\ u_{l}\end{array}\right) \in F^{l} \quad, \quad\left(\begin{array}{c}u_{1} \\ u_{2} \\ \vdots \\ u_{l}\end{array}\right)$ can be linearly presented by $\left(\begin{array}{c}q_{1} \\ q_{1}^{2} \\ \vdots \\ q_{1}^{l}\end{array}\right),\left(\begin{array}{c}q_{2} \\ 2 q_{1}^{2} \\ \vdots \\ l q_{1}^{l}\end{array}\right),\left(\begin{array}{c}q_{2} \\ 2^{2} q_{1}^{2} \\ \vdots \\ l^{2} q_{1}^{l}\end{array}\right), \cdots,\left(\begin{array}{c}q_{l} \\ 2^{l-1} q_{1}^{2} \\ \vdots \\ l^{l-1} q_{1}^{l}\end{array}\right)$.

So $\left\{u_{n}\right\}$ can be linear presented by $\left\{q_{1}^{n}\right\},\left\{n q_{2}^{n}\right\},\left\{n^{2} q_{1}^{n}\right\}, \cdots,\left\{n^{l-1} q_{1}^{n}\right\}$, so $\mathrm{V}$ is the $l$ dimensional vector space on $\mathrm{F}$, and $\left\{q_{1}^{n}\right\},\left\{n q_{2}^{n}\right\},\left\{n^{2} q_{1}^{n}\right\}, \cdots,\left\{n^{l-1} q_{1}^{n}\right\}$ is one set of base of $\mathrm{V}$.

Because $\left\{u_{n}\right\}$ can be linear presented by $\left\{q_{1}^{n}\right\},\left\{n q_{2}^{n}\right\},\left\{n^{2} q_{1}^{n}\right\}, \cdots,\left\{n^{l-1} q_{1}^{n}\right\}$, so $u_{n}=\sum_{k=1}^{l} b_{k} n^{k-1} q_{1}^{n}$, and according to the initial condition $u_{1}, u_{2}, \cdots, u_{l}$, we can confirm $b_{1}, b_{2}, \cdots, b_{l}$, so $u_{n}=\sum_{k=1}^{l} b_{k} n^{k-1} q_{1}^{n}$.

Theorem 1: suppose $V=\left\{\left\{u_{n}\right\} \mid u_{n}=\sum_{k=1}^{l} a_{k} u_{n-k}, a_{k} \in F(k=1,2, \cdots, l), n \geq l+1\right\}$ and $\mathrm{V}$ about Regulation (1) and (2) in theorem 2 is the vector space on the number field $\mathrm{F}$, and $x^{l}=\sum_{k=1}^{l} a_{k} x^{k-1}$ has roots $q_{1}, q_{2}, \cdots, q_{t}$ in which both ones are different and $q_{j}$ is $k_{j}$ multiple root $\left(k_{j} \geq 1\right)$ and $k_{1}+k_{2}+\cdots+k_{t}=l$, so

(1) $\mathrm{V}$ is $l$ dimensional vector space on $\mathrm{F}$

(2) $\left\{q_{1}^{n}\right\},\left\{n q_{1}^{n}\right\},\left\{n^{2} q_{1}^{n}\right\}, \cdots,\left\{n^{k_{1}-1} q_{1}^{n}\right\},\left\{q_{2}^{n}\right\},\left\{n q_{2}^{n}\right\},\left\{n^{2} q_{2}^{n}\right\}, \cdots,\left\{n^{k_{2}-1} q_{2}^{n}\right\}, \cdots \cdots\left\{q_{t}^{n}\right\},\left\{n q_{t}^{n}\right\},\left\{n^{2} q_{t}^{n}\right\}, \cdots,\left\{n^{k_{t}-1} q_{t}^{n}\right\}$ is a set of base of $\mathrm{V}$

(3) Suppose $u_{n}=\sum_{j=1}^{t} \sum_{i=1}^{k_{j}} b_{i j} n^{i-1} q_{j}^{n}$, so according to the initial condition, the values of $u_{1}, u_{2}, \cdots, u_{l}$, we can 
$\operatorname{confirm} b_{i j}\left(i=1, \cdots, k_{j} ; j=1, \cdots, t\right)$ and obtain the recurrence relations $u_{n}=\sum_{j=1}^{t} \sum_{i=1}^{k_{j}} b_{i j} n^{i-1} q_{j}^{n}$.

Example 1: Suppose $x_{1}=1, x_{2}=2, x_{3}=3, x_{n}=\frac{1}{3}\left(x_{n-1}+x_{n-2}+x_{n-3}\right)(n \geq 4)$, find out $\lim _{n \rightarrow \infty} x_{n}$.

Solution: Suppose $V=\left\{\left\{u_{n}\right\} \mid u_{n}=\frac{1}{3}\left(u_{n-1}+u_{n-2}+u_{n-3}\right), n \geq 4\right\}$, so V composes the three-dimensional vector space on the established number field.

From $q^{3}=\frac{1}{3}\left(q^{2}+q+1\right)$, we can obtain $3 q^{3}=q^{2}+q+1$, solve it we can obtain $q_{1}=1, q_{2}=\frac{1}{3}(-1+\sqrt{2} i)$ and $q_{3}=\frac{1}{3}(-1-\sqrt{2} i)$, so $\left\{q_{1}^{n}\right\},\left\{q_{2}^{n}\right\},\left\{q_{3}^{n}\right\}$ are linearly independent, and $\left\{q_{1}^{n}\right\},\left\{q_{2}^{n}\right\},\left\{q_{3}^{n}\right\}$ is one set of base of V, and $\left\{x_{n}\right\}$ can be linearly presented by $\left\{q_{1}^{n}\right\},\left\{q_{2}^{n}\right\},\left\{q_{3}^{n}\right\}$, and

$$
\begin{aligned}
& x_{n}=a q_{1}^{n}+b q_{2}^{n}+c q_{3}^{n} \\
&=a+b\left(\frac{-1+\sqrt{2} i}{3}\right)^{n}+c\left(\frac{-1-\sqrt{2} i}{3}\right)^{n} \\
& \lim _{n \rightarrow \infty} x_{n}=\lim _{n \rightarrow \infty} \frac{1}{3}\left(x_{n-1}+x_{n-2}+x_{n-3}\right) \\
&=\lim _{n \rightarrow \infty} \frac{1}{3}\left\{\left[a+b\left(\frac{-1+\sqrt{2} i}{3}\right)^{n-1}+c\left(\frac{-1-\sqrt{2} i}{3}\right)^{n-1}\right]\right. \\
& \quad+\left[a+b\left(\frac{-1+\sqrt{2} i}{3}\right)^{n-2}+c\left(\frac{-1-\sqrt{2} i}{3}\right)^{n-2}\right] \\
&\left.\quad+\left[a+b\left(\frac{-1+\sqrt{2} i}{3}\right)^{n-3}+c\left(\frac{-1-\sqrt{2} i}{3}\right)^{n-3}\right]\right\}
\end{aligned}
$$

And because $\left\{\begin{array}{l}1=a+b\left(\frac{-1+\sqrt{2} i}{3}\right)+c\left(\frac{-1-\sqrt{2} i}{3}\right) \\ 2=a+b\left(\frac{-1+\sqrt{2} i}{3}\right)^{2}+c\left(\frac{-1-\sqrt{2} i}{3}\right)^{2} \\ 3=a+b\left(\frac{-1+\sqrt{2} i}{3}\right)^{3}+c\left(\frac{-1-\sqrt{2} i}{3}\right)^{3}\end{array}\right.$, solve it, we can obtain $a=\frac{14}{6}$, i.e. $\lim _{n \rightarrow \infty} x_{n}=\frac{14}{6}$.

Example 2: The number sequence, " $2,3,5,10,21 \ldots$, fulfills $x_{n}=4 x_{n-1}-5 x_{n-2}+2 x_{n-3}(n \geq 4)$, find out $\lim _{n \rightarrow \infty} \frac{x_{n}}{x_{n+1}}$.

Solution: Suppose $V=\left\{\left\{u_{n}\right\} \mid u_{n}=4 u_{n-1}-5 u_{n-2}+2 u_{n-3}, n \geq 4\right\}$, so V composes the three-dimensional vector space on the established number field.

From $q^{3}=4 q^{2}-5 q+2$, we can obtain $q_{1}=q_{2}=1, q_{3}=2$.

Because $q_{1}=q_{2} \neq q_{3}$, so $\left\{q_{1}^{n}\right\},\left\{2 q_{1}^{n}\right\},\left\{q_{3}^{n}\right\}$ are linearly independent, and $\left\{q_{1}^{n}\right\},\left\{2 q_{1}^{n}\right\},\left\{q_{3}^{n}\right\}$ is one set of base of V, and $\left\{x_{n}\right\}$ can be linearly presented by their linear combination, i.e. $x_{n}=a q_{1}^{n}+b\left(2 q_{1}^{n}\right)+c q_{3}^{n}$.

From initial condition, we can obtain $\left\{\begin{array}{l}a+2 b+c=2 \\ a q_{1}+2 b q_{1}+c q_{3}=3 \\ a q_{1}^{2}+2 b q_{1}^{2}+c q_{3}^{2}=5\end{array}\right.$, i.e. $\left\{\begin{array}{l}a+2 b+c=2 \\ a+2 b+2 c=3 \\ a+2 b+4 c=5\end{array}\right.$, solve it, we can get $\left\{\begin{array}{l}a+2 b=1 \\ c=1\end{array}\right.$. 


$$
\begin{aligned}
& x_{n}=a q_{1}^{n}+b\left(2 q_{1}^{n}\right)+q_{3}^{n} \quad \lim _{n \rightarrow \infty} \frac{x_{n}}{x_{n+1}}=\lim _{n \rightarrow \infty} \frac{q_{1}^{n}+q_{3}^{n}}{q_{1}^{n+1}+q_{3}^{n+1}} \\
& \text { So, } \begin{aligned}
& =(a+2 b) q_{1}^{n}+q_{3}^{n} \\
& =q_{1}^{n}+q_{3}^{n}
\end{aligned} \text {, and } \quad=\lim _{n \rightarrow \infty} \frac{1+2^{n}}{1+2^{n+1}} \\
& =\frac{1}{2}
\end{aligned}
$$

In conclusion, it is hard to use the method of calculus to find the limit for some recurrence relations, but if we consider them from the direction of the vector space theory, the problem will be effectively solved. In this article, we conclude the solutions to find the limit for the recurrence relations with single root, multiple root and several multiple roots, and the key is to find out a set of proper base, so the recurrence relations can be found according to the initial condition.

\section{References}

Mi, Yongsheng \& Mi, Dongmei. (2002). Application of the High Algebra Method Problem to Maths Analysis. Journal of Changchun University. No.15(2). p.66-69.

Song, Laimin. (2004). Application of the Method of Linear Algebra in Limit. Journal of Tongling College. No. 2. p.100-101. 